Notfall Rettungsmed 2013 • 16:590-592

DOI 10.1007/s10049-013-1736-4

Online publiziert: 7. November 2013

c) Springer-Verlag Berlin Heidelberg 2013

H.-D. Lippert ${ }^{1}$ B B. Gliwitzky ${ }^{2}$

${ }^{1}$ Institut für Rechtsmedizin, Ulm

${ }^{2}$ Deutscher Berufsverband Rettungsdienst e.V.(DBRD), Offenbach an der Queich

\title{
Das Gesetz über den Beruf des Notfallsanitäters
}

\section{Es tut sich etwas im Rettungswesen}

\begin{abstract}
Wäre der Rettungsdienst ein für Deutschland systemrelevanter Bereich, dann wäre das NotSanG innerhalb von $24 \mathrm{~h}$ durch die gesetzgebenden Gremien geschleust und in Kraft gesetzt worden. Da dies aber nicht so ist, hat das Gesetz 24 Jahre auf sich warten lassen. Nahezu gleichzeitig ist das Patientenrechtegesetz in Kraft getreten. Seine Auswirkungen auf das Rettungswesen werden vermutlich noch unterschätzt. Deshalb soll auch von diesem Gesetz die Rede sein. Auch Patientenverfügungen spielen zunehmend eine Rolle im Rettungswesen. Deshalb werden auch ihre Auswirkungen in die Betrachtung der neuen gesetzlichen Regelungen im Rettungswesen aufgezeigt. Die Beiträge dieses Schwerpunkts seien Prof. Dr. Friedrich Wilhelm Ahnefeld gewidmet, der sich wie kaum ein anderer um das Rettungswesen in Deutschland verdient gemacht hat.
\end{abstract}

Viele Jahre wurde eine Novellierung des Rettungsassistentengesetzes von allen Beteiligten in der Notfall- und Rettungsmedizin dringend gefordert, um den steigenden Ansprüchen an den Beruf gerecht zu werden und den Problemen des jetzigen Rettungsassistentengesetzes entspre- chend zu begegnen. ${ }^{1}$ Die Zeitschrift Notfall + Rettungsmedizin widmet in diesem Heft diesem Thema einen Schwerpunkt. Ausgewiesene Fachleute haben in dieser Ausgabe der Notfall + Rettungsmedizin für Sie als Leser insgesamt 4 Arbeiten entsprechend aufbereitet, um die wesentlichen medizinischen und juristischen Aspekte des neuen Gesetzes und die damit verbundenen Veränderungen darzustellen. Wir möchten an dieser Stelle als Herausgeber dieses Leitthemenhefts ganz besonders daran erinnern, dass es Ahnefeld war, der bereits in den 70er Jahren eine dreijährige Berufsausbildung für Rettungssanitäter auf gesetzlicher Grundlage gefordert hatte. Über 30 Jahre nach den ersten Bemühungen um eine qualifizierte Ausbildung liegt das Gesetz über die Notfallsanitäterin und über den Notfallsanitäter nun vor. ${ }^{2}$ Leider hat der Nestor der deutschen Notfallmedizin das Inkrafttreten des NotSanG nicht mehr erleben dürfen. Er wäre sicher stolz gewesen, dass seine Beharrlichkeit doch noch zum Ziel geführt hat.

\footnotetext{
${ }^{1}$ Gesetz vom 30.06. 1984 BGBI. I S. 1384. Vgl. hierzu auch Ruppert M, Reeb R, Ufer MR, Stratmann D, Altemeyer KH (2002) Personal im Rettungsdienst: Brauchen wir neue Konzepte? Notfall + Rettungsmed 2002 (5) 375-379; in diesem Zusammenhang darf auch das Reisensburger Memorandum zum RettAssG nicht unerwähnt bleiben. Es findet sich - auch heute noch lesenswert - in Gorgaß, Ahnefeld, Rettungsassistent und Rettungssanitäter, 6 . Aufl. 2001, Kap. 38.5.

${ }^{2}$ Gesetz vom 22.05.2013 BGBI. I S. 1348.
}

\section{Notfallsanitätergesetz - Was ändert sich gegenüber dem Rettungsassistentengesetz?}

Mit dem Notfallsanitätergesetz ist nicht nur einfach das Rettungsassistentengesetz novelliert worden, vielmehr wurde ein nun deutlich attraktiverer und neuer Ausbildungsberuf konzipiert, der den Anforderungen an ein modernes Berufsbild gerecht wird. Zentrale Forderungen aller an der Diskussion Beteiligten, wie die dreijährige alternierende Ausbildung, die Vergütung der Auszubildenden, das Examen am Ende der Ausbildung sowie die klare Beschreibung des Ausbildungsziels wurden entsprechend durch den Gesetzgeber berücksichtigt. Gerade das Ausbildungsziel ist nun wesentlich konkreter formuliert, als dies bis dato im RettAssG der Fall war. Dies bedeutet natürlich auch eine damit verbundene erhöhte Rechtssicherheit für die Notfallsanitäter bei der Durchführung von sog. invasiven Maßnahmen. Eine undifferenzierte einfache Überleitung von jetzigen Rettungsassistenten wie beim Inkrafttreten des Rettungsassistentengesetzes 1989 wurde durch den Gesetzgeber ausdrücklich durch entsprechende Regelungen im Gesetz $₫ 32$ verhindert. Jetzige Rettungsassistenten müssen eine Ergänzungsprüfung und - je nach Berufserfahrung - eine Ergänzungsschulung absolvieren. Dies stellt ein erhebliches Qualitätsmerkmal des Gesetzes dar. Am Ende der Ausbildung werden somit hoffentlich kompetente ausgebildete Notfallsanitäter die Schulen verlassen, die sowohl eigen- 
verantwortlich, aber auch im Team mit dem Notarzt, für eine noch bessere $\mathrm{Pa}$ tientenversorgung sorgen werden.

\section{Patientenrechtegesetz - Auswirkungen auf die Behand- lung von Notfallpatienten?}

Von den Protagonisten des NotSanGs eher unbeachtet hat sich eine neue Vorschrift ins BGB eingeschlichen, deren Neuerungen auch im Rettungswesen Auswirkungen zeigen werden. Durch das Patientenrechtegeset $z^{3}$ ist der Behandlungsvertrag als spezieller Dienstvertrag neu ins BGB eingefügt worden ( $\$ \$ 630 \mathrm{a}$ ff. BGB). Bisher unterlag der Arzt-Patienten-Vertrag den Regeln des allgemeinen Dienstvertrags nach $\$ \$ 611 \mathrm{ff}$. BGB. Dahinter verbirgt sich nicht lediglich die gesetzliche Regelung des Arzt-Patienten-Verhältnisses, sondern jede Art von Behandlung. Das heißt, auf das Rettungswesen, also den Rettungs- und den Notarztdienst bezogen, ist dieses Gesetz ab sofort anwendbar, auch wenn es noch gar keine Notfallsanitäter gibt. Vom Patientenrechtegesetz ausgenommen ist im Rettungswesen lediglich der bewusstlose Notfallpatient, weil mit ihm bekanntlich kein wirksamer Vertrag abgeschlossen wird. Hier verbleibt es bei den Regeln der Geschäftsführung ohne Auftrag wie bisher auch.

Findet das Patientenrechtegesetz auf das Rettungswesen Anwendung, dann heißt dies, dass zwischen dem Patienten und dem Träger des Rettungsdienstes und dem des Notarztdienstes (je nach Organisationsform) ein Behandlungsvertrag mit jeweils spezifischem Inhalt zustande kommt. Auf den Vertragsschluss sind die allgemeinen Vorschriften des BGB über Verträge anzuwenden. Das heißt, die Vertragspartner müssen geschäftsfähig sein (oder bei Geschäftsunfähigen gesetzlich vertreten sein). Die Einwilligungsfähigkeit, die für die Einwilligung in das Behandlungsgeschehen ausreichend sein mag ( $\$ \$ 630 \mathrm{~d}$ BGB), reicht dafür nicht aus. Das eingesetzte Personal der Träger (Rettungsassistent, Notfallsanitäter und/ oder Notarzt) sind in diesem Vertrag lediglich dessen Erfüllungsgehilfen, nicht aber Vertragspartner. Die Träger müs-

${ }^{3}$ Gesetz vom 20.02. 2013 BGBI. I S. 277. sen allerdings nach dem Patientenrechtegesetz sicherstellen, dass einwilligungsfähige Patienten informiert und aufgeklärt werden und in die Behandlung einwilligen. Ferner ist das Behandlungsgeschehen zu dokumentieren. Wie auch immer man zu den Regeln der Beweislastverteilung in $\$ 630 \mathrm{~h}$ für Behandlungs- und Aufklärungsfehler stehen $\mathrm{mag}^{4}$, sie finden auf den Behandlungsvertrag Anwendung und können für den Behandler erhebliche Nachteile zur Folge haben, wenn die darin verankerten Vermutungen von der Behandlerseite nicht widerlegt werden können. Es gilt v. a., dies für den Behandlungsfehler zu bedenken.

Ansonsten kann das vom Patienten vertraglich geschuldete Entgelt für den Rettungseinsatz nur gefordert werden, wenn dieser vereinbarungsgemäß aufgrund eines wirksamen Vertrags erfüllt wurde. Kürzungen sind daher denkbar und möglich. Die Schlechterfüllung des Vertrags, etwa bei der Schädigung des Patienten, kann zudem Schadenersatzansprüche zur Folge haben. Alternativ kann hier wie bisher auch die unerlaubte Handlung als Anspruchsgrundlage herangezogen werden.

\section{Patientenverfügungen und der Wille des Patienten}

Im Rettungswesen, in dem eine gewisse Zahl von Patienten bewusstlos und damit nicht imstande ist, in die notwendige Behandlung einzuwilligen, gewinnt $\$ 630$ d BGB eine besondere Bedeutung. Dort ist festgelegt, dass beim bewusstlosen, also nicht einwilligungsfähigen $\mathrm{Pa}$ tienten die Einwilligung des Berechtigten einzuholen ist. Dies heißt konkret, dass Eltern für ihre Kinder einwilligen und der Betreuer dies für den Betreuten tun muss. Liegt eine Patientenverfügung vor, so ist nach $₫$ 1901a BGB zu verfahren. Dies bedeutet, dass nach dem Vorliegen der Patientenverfügung gefragt werden muss, sofern sie für den vorliegenden Fall

\footnotetext{
${ }^{4}$ Vgl. hierzu z. B. Rehborn, Das Patientenrechtegesetz, GesR 2013, 257, Katzenmeier, NJW 2013, 817; Jaeger, Patientenrechtegesetz, 2013, $\S 630$ h Rz. 337 ff. m.w. Nachw. um nur einige zu nennen. Völlig ablehnend Mäsch: Demokratisches Schamanentum in Wahlkampfzeiten, NJW 2013,1354 (passt nicht ins Vertragsrecht).
}

der Erkrankung Aussagen trifft. Fehlt eine solche Verfügung oder trifft die vorliegende auf den aktuellen Sachverhalt nicht zu, so haben die Behandler den mutmaßlichen Willen des Patienten zu ermitteln, ggf. durch Befragung naher Angehöriger oder Bezugspersonen, auch des Betreuers, sofern dies ohne Verzögerung der erforderlichen Behandlung möglich ist. Duldet die erforderliche Behandlungsmaßnahme keinen Aufschub, ohne dass der Patient einen weiteren Schaden erleidet, so darf behandelt werden (1904 Abs. 1 BGB).

Liegt eine Patientenverfügung vor und trifft sie auf den vorliegenden Sachverhalt $\mathrm{zu}$, so ist sie zu beachten. In diesem Fall hat der Behandler die gewünschten Maßnahmen durchzuführen oder auch eine Behandlung zu unterlassen, selbst wenn er sie für erforderlich hält. ${ }^{5}$ Mit $\$ 630 \mathrm{~d}$ BGB hat der Gesetzgeberfür das Rettungswesen eine hohe Hürde errichtet, die es im Rettungswesen handhabbar zu machen gilt, da die Pflicht zur Dokumentation auch diesen Aspekt umfasst.

\section{Umsetzung der neuen Gesetze in die Praxis}

Eine herausragende Rolle bei der Umsetzung des Notfallsanitätergesetzes werden die Schulen, die Kliniken, die Lehrrettungswachen sowie die Ärztlichen Leiter Rettungsdienste spielen. Nur wenn hier in den nächsten Jahren die notwendigen Schritte eingeleitet werden, wird am Ende ein hochkompetenter Fachmann für den Rettungsdienst zur Verfügung stehen, der eine hochwertige eigenverantwortliche und teamorientierte Notfallversorgung leisten kann. Brokmann et al. gehen in einem der Artikel dieser wichtigen Sache nach. Um insbesondere die Diskussionen um die Kompetenzen zu versachlichen, sind alle Beteiligten aufgefordert, einen möglichst verbindlichen Katalog an Maßnahmen zu entwickeln, der ge-

\footnotetext{
${ }^{5}$ So im Ergebnis auch neuestens Boehmke, Unterlassen lebensrettender Maßnahmen bei einwilligungsunfähigen Patienten, NJW 2013, 1412. Im Bereich der Notfallmedizin, wo die Entscheidung über die Behandlung oft unter zeitlichem Druck zu treffen ist, dürfte die sonst zutreffende Forderung nach der Bestellung eines Betreuers zumeist nicht zu erfüllen sein. Also wird im Zweifel behandelt werden.
} 
eignet ist, dem klar formulierten Ausbildungsziel im Notfallsanitätergesetz Rechnung zu tragen. Dieser muss sich an den Leitlinien der Fachgesellschaften orientieren und regelmäßig den Veränderungen in der Akutmedizin angepasst werden. Es ist geboten, dass unnötige Grabenkämpfe zwischen den Berufsgruppen vermieden werden und das der Blick stärker auf die wichtigste Person in der Notfallmedizin gelenkt wird: Den Notfallpatienten. Dieser profitiert sicherlich von besser qualifizierterem Personal.

Das Notfallsanitätergesetz bietet viele Chancen. Die zahlreiche Kritik aus den Reihen der Ärzteschaft, es würde mit dem Notfallsanitätergesetz der Einstieg in ein Paramedic System versucht, ist aus Sicht der Autoren unbegründet. ${ }^{6}$ Auch die immer wieder geäußerte Sorge um die Patientensicherheit ist nicht immer nachvollziehbar. ${ }^{7}$ Besser ausgebildetes Personal steigert eher die Patientensicherheit, als dass es sie schwächt. Es geht in den nächsten Jahren viel mehr darum, den steigenden Anforderungen und den sich verändernden Gegebenheiten (demographischer Wandel, Zunahme des Einsatzaufkommens etc. $)^{8}$ adäquat zu begegnen. Dazu bedarf eines differenzierteren Einsatzes der zur Verfügung stehenden Ressourcen. Ganz sicher braucht es neben kompetenten Notfallsanitätern aber auch weiterhin kompetente Notärzte.

Erhebliche Anstrengungen bei der Umsetzung erfordern die Vorschriften des Patientenrechtegesetzes und der bereits schon länger geltenden Vorschriften über die Patientenverfügung im BGB selbst dann, wenn in weiten Teilen dadurch nur die bereits bisher geltende Rechtslage in Gesetzesform gegossen worden ist. Hier

\footnotetext{
${ }^{6}$ Stellungnahme der BAND zum Referentenentwurf der Bundesregierung zum Entwurf zum Beruf der Notfallsanitäterinund des Notfallsanitäters. http://www.band-online.de/Stellungnahmen_und_Empfehlungen_32.html abgerufen am 09.06.2013;Stellungnahme der Bundesärztekammer. http://www.bundesaerztekammer. de/downloads/StellBAeK_NotSanG_21062012. pdf abgerufen am 09.06.2013.

${ }^{7}$ Stellungnahme BAND, Stellungnahme Bundesärztekammer (Fn. 6).

${ }^{8}$ Koch B, Wendt M, Lackner CK, Ahnefeld FW (2008) Herausforderungen an die Notfallversorgung der Zukunft:„Regional Health Care“ (RHC) Notfall Rettungsmed 11:491-499.
}

gilt der alte Spruch, wonach der Teufel bekanntlich im Detail steckt. Sie ins Bewusstsein der Verantwortlichen zu rücken, ist das Ziel dieser Zeilen. ${ }^{9}$

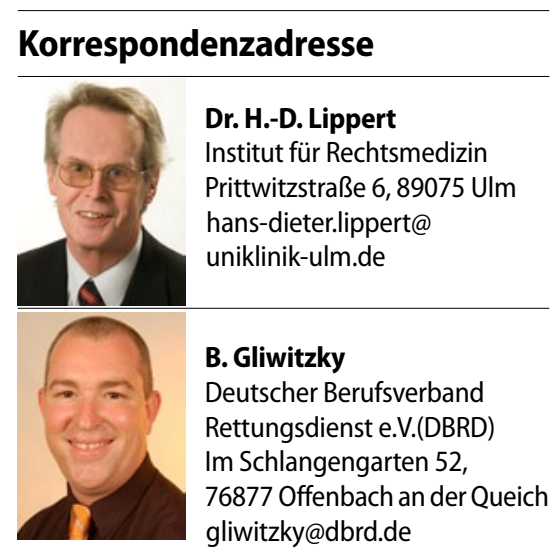

\section{Einhaltung der ethischen Richtlinien}

Interessenkonflikt. H.D. Lippert gibt an, dass kein Interessenkonflikt besteht. B. Gliwitzky ist Vorstandsmitglied des Deutschen Berufsverbandes Rettungsdienst e.V (DBRD) in Offenbach an der Queich und Geschäftsführender Gesellschafter von MegaMed Notfallmanagement in Annweiler.

Dieser Beitrag beinhaltet keine Studien an Menschen oderTieren

\footnotetext{
${ }_{9}^{9}$ Auf die weitere Änderung des BGB in§1906, soweit die zwangsweise Behandlung Einwilligungsunfähiger betroffen ist, soll an dieser Stelle nicht eingegangen werden, da die Notfallmedizin davon eher selten betroffen sein dürfte. Der Hinweis erfolgt nur der Vollständigkeit halber.
}

Weitere Infos auf springermedizin.de

Dossier

Deutscher Kongress für Orthopädie und Unfallchirurgie (DKOU) 2013

Wir waren vor Ort und haben das wichtigste für Sie in unserem Kon gressdossier zusammen getragen Von Fallstricken in der Osteoporosediagnostik über den neuesten Stand der Knieendoprothetik bis hin zur Frage, ob Deutschland bei orthopädischen Eingriffen überversorgt ist.

Alle Beiträge und Videos finden Sie unter

- www.springermedizin.de/ dkou-2013 